IdeAs

Idées d'Amériques

13 | 2019

La photographie documentaire contemporaine dans les Amériques

\title{
L'élection de Bolsonaro, la politique étrangère brésilienne en 2019 et l'avenir de la coopération sud-Sud
}

Renata Albuquerque Ribeiro et Carlos R. S. Milani

\section{OpenEdition}

Journals

Édition électronique

URL : http://journals.openedition.org/ideas/5578

DOI : $10.4000 /$ ideas.5578

ISSN : 1950-5701

Éditeur

Institut des Amériques

Référence électronique

Renata Albuquerque Ribeiro et Carlos R. S. Milani, « L'élection de Bolsonaro, la politique étrangère brésilienne en 2019 et l'avenir de la coopération Sud-Sud », IdeAs [En ligne], 13 | 2019, mis en ligne le 01 mars 2019, consulté le 21 avril 2019. URL : http://journals.openedition.org/ideas/5578 ; DOI :

10.4000/ideas.5578

Ce document a été généré automatiquement le 21 avril 2019

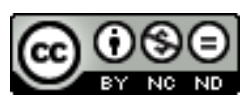

IdeAs - Idées d'Amériques est mis à disposition selon les termes de la licence Creative Commons Attribution - Pas d'Utilisation Commerciale - Pas de Modification 4.0 International. 


\title{
L'élection de Bolsonaro, la politique étrangère brésilienne en 2019 et l'avenir de la coopération Sud-Sud
}

\author{
Renata Albuquerque Ribeiro et Carlos R. S. Milani
}

1 En termes de politique étrangère, l'élection de Jair Bolsonaro présageait un changement important en ce qui concerne les stratégies économiques, commerciales, multilatérales et géopolitiques. Durant les cent premiers jours du gouvernement Bolsonaro, des tensions entre différentes coalitions de soutien (forces armées, acteurs du système judiciaire, églises néo-pentecôtistes, entourage proche du président, etc.) ont émergé, mettant en relief des orientations peu convergentes dans différents domaines d'action publique, dont le développement stratégique, l'intégration économique et la politique internationale. En dépit de ces tensions internes, l'arrivée au pouvoir de Bolsonaro met en exergue de nouvelles perceptions et des perspectives de changement importantes pour l'action du Brésil à l'international. Quels sont les principaux objectifs de la politique étrangère brésilienne (PEB) à partir de 2019 ? Quels scénarios peut-on envisager? Qu'adviendra-t-il plus particulièrement de la coopération Sud-Sud, qui avait été activement développée par les gouvernements Lula puis, dans une moindre mesure, sous Dilma Rousseff ? Cet article tente de répondre à ces questions en présentant les principaux scénarios pouvant être attribués à la politique étrangère au sein du gouvernement Bolsonaro, puis en mettant l'accent sur les perspectives de coopération Sud-Sud.

2 Le programme électoral de Bolsonaro mentionnait très timidement le thème de la politique étrangère, soulignant l'abandon d'une prétendue «idéologisation » et défendant une alliance forte avec les États-Unis et la signature d'accords commerciaux bilatéraux. Dès le 14 novembre 2018, le président élu dévoilait le nom de son nouveau ministre des Affaires étrangères: l'ambassadeur Ernesto Araújo. Cette nomination a suscité de nombreuses réactions, y compris parmi les diplomates. Certains ont dénoncé une rupture hiérarchique, le diplomate n'ayant jamais dirigé de mission à l'étranger et n'ayant été promu ambassadeur qu'en 2018 (Skodowski T., 2018). Un autre enjeu concerne la logique de cette nomination. Le nom d'Araújo aurait été suggéré par le fils du président, Eduardo 
Bolsonaro (Folhapress, 2018), lui-même intéressé par les questions internationales, sous l'influence d'Olavo de Carvalho. La troisième critique souligne les pratiques atypiques du nouveau ministre. Durant la campagne électorale de 2018, celui-ci a commenté à souhait et de manière ouvertement militante, via son blog, des enjeux de politique intérieure et étrangère : « la foi dans le Christ signifie, aujourd'hui, de lutter contre le globalisme, dont l'objectif ultime est de briser la connexion entre Dieu et l'homme» $(22 / 09 / 2018)$; «le climatisme - idéologie du changement climatique - n'est qu'une tactique globaliste pour instituer la peur et obtenir plus de pouvoir » (12/10/2018), etc ${ }^{1}$.

Durant les cent premiers jours au pouvoir, le nouveau gouvernement a déjà exposé certaines composantes de la nouvelle politique étrangère. Certains points méritent une attention particulière. Le premier est l'alignement clair avec les États-Unis. Les marges d'action du Brésil de Bolsonaro dans le scénario international seraient en accord avec la conception d'un axe du pouvoir trumpiste, élaboré par Steve Bannon, qui inclut, outre le Brésil, Israël, l'Italie et la Hongrie. Après les résultats des élections, Bolsonaro et Araújo ont d'ailleurs entamé un rapprochement inédit avec Israël. Cette stratégie peut néanmoins s'avérer coûteuse pour le Brésil. Par exemple, en novembre 2018, la délégation égyptienne a annulé la visite officielle du ministre des Affaires étrangères du Brésil, Aloysio Nunes, à la suite de la déclaration controversée de Bolsonaro sur le transfert de l'ambassade du Brésil de Tel Aviv à Jérusalem. Cette déclaration a suscité un vif mécontentement dans les pays arabes, avec des effets potentiellement négatifs pour les exportations brésiliennes de viande et de volaille (Boadle A., 2018). La décision de se rapprocher des pays avec lesquels le gouvernement actuel a une proximité idéologique répond également aux préoccupations des secteurs évangéliques - un noyau de soutien interne important $\mathrm{du}$ président Bolsonaro. Cette décision est pourtant en nette contradiction avec la promesse électorale de "désidéologiser la politique étrangère ". Dans le même sens de convergence idéologique, il est possible d'anticiper un rapprochement au niveau régional avec la Colombie de I. Duque et le Paraguay de M. Abdo. La célébration du Sommet conservateur des Amériques, qui s'est tenu en décembre 2018 à Foz do Iguaçu (Brésil), a compté avec la participation de représentants conservateurs de plusieurs pays de la région, mettant en évidence la construction d'un réseau transnational conservateur régional. Le ministre Araújo s'en est d'ailleurs pris explicitement à Cuba et au Venezuela lors de l'investiture de Bolsonaro, le $1^{\text {er }}$ janvier 2019. S'il s'agissait de satisfaire l'électorat conservateur et les militants anti-PT, ce discours a été qualifié de " gaffe diplomatique » par certains journalistes. Il a aussi été critiqué par certains secteurs de son ministère, qui cherchent à préserver la tradition diplomatique pacifiste du Brésil, indépendamment de l'orientation politique des gouvernements.

4 Enfin, deux autres épisodes méritent une mention spéciale dans l'analyse de la PEB : l'évolution des relations avec la Chine et la perte de capital diplomatique dans le domaine multilatéral. Le gouvernement chinois, principal partenaire commercial du Brésil, a déjà exprimé sa préoccupation face aux déclarations agressives du chancelier et de l'un des fils du président, Eduardo Bolsonaro. En ce qui concerne les négociations multilatérales, le Brésil est considéré comme un acteur mondial et un bâtisseur de ponts (bridge-builder), particulièrement dans le domaine climatique. Bolsonaro a pourtant déjà déclaré que le Brésil pourrait quitter l'Accord de Paris (2015), voire même les Nations unies. Le ministre Ernesto Araújo s'est dit quant à lui convaincu que le changement climatique constitue une « conspiration marxiste » (Watts J., 2018) et que le « mondialisme » est contraire aux 
intérêts de l'Occident chrétien. Indépendamment de la décision finale par rapport à l'Accord de Paris, le fait est que le Brésil a décidé de ne pas accueillir la Conférence des parties à l'accord des Nations unies sur le climat (COP 25), prévue fin 2019. La migration est un autre domaine du multilatéralisme pour lequel le nouveau gouvernement suscite des interrogations : l'administration Bolsonaro a indiqué que le Brésil devrait se retirer du Pacte mondial sur les migrations, signé en décembre 2018 par le gouvernement Temer (Deutsche Welle, 2019). Ce fut l'une des premières mesures du nouveau gouvernement en matière de politique étrangère.

5 Face à un ensemble de décisions (et de contre-décisions) qui semblent traduire une faible cohérence stratégique, la branche militaire du gouvernement, principalement par le biais des déclarations du vice-président, le général Hamilton Mourão, a tenté de maintenir la rationalité stratégique de la politique étrangère, en veillant par exemple à ce que le partenariat avec la Chine soit considéré comme fondamental d'un point de vue géoéconomique. Mourão a aussi affirmé que le Brésil ne devrait pas transférer son ambassade en Israël à Jérusalem.

6 Il n'en demeure pas moins que d'énormes incertitudes pèsent sur la pérennité des actions qui avaient permis au Brésil de se faire reconnaître comme un acteur de premier plan dans le système international. Le Brésil avait par exemple joué un rôle décisif pour l'intégration régionale sud-américaine (UNASUR), le renforcement de l'autonomie latinoaméricaine (CELAC), l'affirmation des pays émergents (BRICS, Nouvelle Banque de Développement, forum IBAS), le rapprochement de l'Amérique du Sud avec les pays arabes et africains, etc. Avec une politique étrangère qui se tourne résolument vers les États-Unis de Donald Trump, il est ainsi nécessaire de s'interroger sur l'avenir de la Coopération Sud-Sud (CSS), en partie coordonnée par l'Agence brésilienne de coopération (Milani C., 2017).

7 Un premier enjeu rhétorique et diplomatique concerne la manière de réconcilier les éléments d'un régime symbolique légué par le tiers-mondisme au sein d'un gouvernement qui dénonce le "globalisme » et le "marxisme culturel», selon les mots du chancelier lui-même. Sans négliger les intérêts - économiques, stratégiques et de sécurité - que peut avoir le Brésil à travers la CSS, la rhétorique de solidarité était le fondement moral de justification de cette modalité de coopération établie par le Brésil avec ses partenaires des pays du Sud (Closs M., 2018). Comment justifier les pratiques de la CSS brésilienne sans mettre l'accent sur le discours de solidarité avec le Sud? De manière plus concrète, l'un des mécanismes les plus utilisés par les gouvernements brésiliens précédents (et pas exclusivement ceux du PT) afin de promouvoir ses projets de coopération Sud-Sud a été l'articulation avec les organisations internationales. Sur ce point, Araújo a également marqué son intention d'emprunter une nouvelle voie, « contre tout ce qui avait été construit auparavant ». On peut donc s'attendre à un affaiblissement de la place du Brésil dans les organisations multilatérales (FAO, OMS, PAM, PNUD, etc.), avec des effets directs et indirects pour la coopération Sud-Sud. Soulignons enfin que la politique étrangère répond, en tant que politique publique, à la dynamique des jeux de pouvoir au niveau national. En outre, elle se distingue des autres politiques publiques dans la mesure où elle est sous l'influence directe et systématique des facteurs régionaux et mondiaux (Milani C., 2015). Les « années d'or » de la coopération proposée par le Brésil présentaient, en toile de fond, des facteurs internes et systémiques permettant à ce pays de devenir un rising player dans ce domaine, aux côtés de pays tels que la Chine, l'Inde et l'Afrique du Sud, tout en essayant de construire des règles, critères et procédures qui se 
distinguent de la coopération des pays du Nord, en particulier celle des membres du Comité d'aide au développement de l'OCDE. Le projet de faire adhérer le Brésil à l'OCDE, initié sous le gouvernement Temer, gagne en puissance sous Bolsonaro: en plus de sa proximité avec les États-Unis de Donald Trump, la ligne économique du nouveau gouvernement consiste à approfondir les réformes ultra-libérales initiées par Temer. L'entrée du Brésil à l'OCDE pourrait néanmoins compromettre ses relations avec les pays en développement qui considéraient le Brésil comme un membre important du G-77. Le tableau en annexe résume certains de ces facteurs nationaux et systémiques, ainsi que les scénarios possibles, sous le gouvernement Bolsonaro.

Depuis les résultats des élections, les médias et l'opinion publique ont mis l'accent sur la politique étrangère du Brésil en raison des déclarations controversées du président élu et de son ministre des Affaires étrangères. Les incertitudes sont nombreuses quant à la politique qui sera adoptée, entretenues durant les cent premiers jours du gouvernement Bolsonaro par une série de décisions peu cohérentes. D'un côté, on peut trouver des traces de la politique étrangère de Temer en ce qui concerne le rapprochement avec les États-Unis et la réforme du MERCOSUR. Pour ce qui est des relations avec l'Union européenne, les relations avec les pays les plus puissants du groupe (Allemagne, France) pourraient être perturbées par les affinités idéologiques du Brésil avec les gouvernements actuels de l'Italie et de la Hongrie. En outre, certains pays européens ont déjà laissé entendre qu'ils n'accepteraient pas une remise en cause des engagements du Brésil en faveur des droits de l'homme et du changement climatique (Estado de São Paulo, 2018). Le président français, Emmanuel Macron, a directement lié l'engagement du Brésil dans l'accord de Paris avec la poursuite des négociations entre l'Union européenne avec le MERCOSUR, dans le cadre d'un exercice diplomatique de linkage politics (Stein A., 1980). Dans un tel contexte, la coopération Sud-Sud devrait faire face à un long désert politique, car les principales exigences pour sa mise en œuvre en tant qu'instrument efficace de la politique étrangère sont absentes au sein du gouvernement actuel. Les années Lula et Rousseff ont montré que la CSS était un instrument de la politique étrangère du Brésil susceptible de contribuer à davantage d'autonomie et de développement, non sans contradictions. Les données sur la diversification des marchés et sur l'augmentation significative des exportations brésiliennes vers l'Afrique au cours de cette période confirment cette utilisation stratégique de la CSS. Partant du principe que la solidarité et les intérêts vont de pair dans le domaine de la coopération internationale pour le développement, qu'elle soit Nord-Sud ou Sud-Sud, il reste à savoir si - et comment - le nouveau gouvernement construira une rhétorique et une diplomatie pour justifier les liens historiques entre le Brésil et les pays en développement dans les relations internationales (Milani C., 2018).

\section{BIBLIOGRAPHIE}

Boadle, Anthony, «Liga Árabe diz a Bolsonaro que mudança de embaixada em Israel pode prejudicar relações », Reuters, 10 décembre 2018. URL : https://extra.globo.com/noticias/brasil/ 
liga-arabe-diz-bolsonaro-que-mudanca-de-embaixada-em-israel-pode-prejudicar-

relacoes-23295724.html

Closs, Marília, « Parceiros ou "mulambos" ? A Cooperação Sul-Sul e as eleições. Iesp nas eleições », 04 octobre 2018. URL : http://iespnaseleicoes.com.br/parceiros-ou-mulambos-acooperacao-sul-sul-e-as-eleicoes/.

Deutsche Welle, «Brasil deixa pacto de migração da ONU », Deutsche Welle, 9 janvier 2019. URL : https://www.dw.com/pt-br/brasil-deixa-pacto-de-migra \%C3 \%A7 \%C3 \%A3o-da-onu/a-47005898

Folhapress, " Escolha de chanceler reforça ambição internacional de Eduardo Bolsonaro ", Folha de São Paulo, 15 novembre 2018. URL : https://www1.folha.uol.com.br/mundo/2018/11/escolhade-chanceler-ernesto-araujo-e-vitoria-de-eduardo-bolsonaro.shtml

Milani, Carlos R. S, ABC 30 anos: história e desafios futuros, Brasília, Agência Brasileira de Cooperação, 2017.

Milani, Carlos R. S, « Política Externa é Política Pública? » Insight Inteligência, Rio de Janeiro, v. XVIII, 2015, p. 56-75.

Milani, Carlos R. S., Solidariedade e Interesse: motivações e estratégias na cooperação internacional para o desenvolvimento, Curitiba, Appris, 2018.

O Estado de São Paulo, « Acordo entre Mercosul e UE fracassa e negociação dependerá de governo Bolsonaro ", 14 décembre 2018. URL : https://economia.estadao.com.br/noticias/geral,acordoentre-ue-e-mercosul-fracassa-e-negociacao-dependera-de-governo-bolsonaroem-2019,70002646460

Skodowski, Thais, «Relações Exteriores : futuro ministro jamais chefiou embaixada », R7, 14 novembre 2018. URL : https://noticias.r7.com/brasil/relacoes-exteriores-futuro-ministrojamais-chefiou-embaixada-14112018

Stein, Arthur A., « The Politics of Linkage », World Politics, vol. 3, n. 1, 1980, p. 62-81.

Watts, Jonathan, « Brazil's new foreign minister believes climate chance is a Marxist plot », The Guardian, 15 novembre 2018. URL : https://www.theguardian.com/world/2018/nov/15/brazilforeign-minister-ernesto-araujo-climate-change-marxist-plot

\section{ANNEXES}

Scénarios possibles pour l'insertion internationale du Brésil sous Bolsonaros

\begin{tabular}{|l|l|l|}
\hline Niveaux & Facteurs & Scénarios \\
\hline
\end{tabular}




\begin{tabular}{|c|c|c|}
\hline \multirow{5}{*}{$\begin{array}{l}\text { Plan } \\
\text { national }\end{array}$} & $\begin{array}{l}\text { Stabilité de la } \\
\text { coalition et du } \\
\text { gouvernement }\end{array}$ & $\begin{array}{l}\text { Difficile et improbable. Le président présente cinq groupes ou } \\
\text { forces sociales qui constituent son noyau d'appui : sa famille, } \\
\text { l'aile économique ultralibérale dirigée par le ministre des } \\
\text { finances (Paulo Guedes); les militaires; des segments } \\
\text { importants de l'appareil judiciaire et du parquet autour du } \\
\text { ministre de la justice (Sérgio Moro); et les évangéliques. Ces } \\
\text { groupes sont des acteurs politiques car ils sont capables de } \\
\text { mobiliser et de créer des agendas à la fois dans le débat public } \\
\text { et au sein de l'État. Cependant, il existe de fortes luttes de } \\
\text { pouvoir entre eux, et ces groupes peuvent adopter des } \\
\text { positions opposées ou, du moins, ayant une faible capacité de } \\
\text { convergence, ce qui pourrait compromettre la stabilité } \\
\text { politique du gouvernement. }\end{array}$ \\
\hline & $\begin{array}{l}\text { Stabilité } \\
\text { économique et } \\
\text { disponibilité } \\
\text { budgétaire pour } \\
\text { la coopération } \\
\text { Sud-Sud }\end{array}$ & $\begin{array}{l}\text { Difficile, mais possible. La stabilité économique est } \\
\text { directement liée à la stabilité politique et à la capacité de ces } \\
\text { groupes d'appui à établir des directives convergentes avec le } \\
\text { Congrès national (réforme du système de sécurité sociale, } \\
\text { révision du statut de désarmement, réforme du système } \\
\text { judiciaire, par exemple). En ce qui concerne plus } \\
\text { particulièrement la coopération sud-Sud, le budget de } \\
\text { l'Agence de coopération brésilienne a connu son apogée en } \\
2010 \text { et a diminué depuis. }\end{array}$ \\
\hline & $\begin{array}{l}\text { Perception de la } \\
\text { nature } \\
\text { stratégique de la } \\
\text { coopération sud- } \\
\text { Sud }\end{array}$ & $\begin{array}{l}\text { Possible. Les déclarations du futur chancelier et du vice- } \\
\text { président montrent le contraire. Cependant, ils peuvent } \\
\text { signaler un partenariat avec l'axe du pouvoir de droite et } \\
\text { d'extrême droite (Steve Bannon), y compris l'Italie et Israël, } \\
\text { ce qui représente un déplacement radical des programmes de } \\
\text { coopération du Sud vers le Nord. Les impacts sur l'Agence } \\
\text { brésilienne de coopération doivent être surveillés en } \\
\text { particulier. }\end{array}$ \\
\hline & $\begin{array}{l}\text { Leadership } \quad \mathrm{du} \\
\text { président }\end{array}$ & $\begin{array}{l}\text { Absent. Le président élu tend à pratiquer une délégation de la } \\
\text { politique étrangère qui néglige la diplomatie présidentielle. } \\
\text { Son discours au Forum économique mondial de Davos } \\
\text { (janvier 2019) était une première illustration de ce } \\
\text { phénomène. }\end{array}$ \\
\hline & $\begin{array}{ll}\text { Participation } & \text { des } \\
\text { acteurs } & \text { non } \\
\text { étatiques } & \end{array}$ & $\begin{array}{l}\text { Difficile. Le contexte évoque un retour à «l'isolement } \\
\text { bureaucratique " de l'Itamaraty ou à la "privatisation de la } \\
\text { politique étrangère ", caractérisé par moins de transparence } \\
\text { et peu d'espace de dialogue avec les organisations de la } \\
\text { société civile. Le décret de réforme de la loi sur l'accès à } \\
\text { l'information (signé par le vice-président en exercice lors de } \\
\text { la visite de Bolsonaro à Davos) va dans le sens de la censure et } \\
\text { d'une reddition de comptes nettement moins importante. }\end{array}$ \\
\hline
\end{tabular}




\begin{tabular}{|c|c|c|}
\hline \multirow[t]{2}{*}{$\begin{array}{l}\text { Plan } \\
\text { systémique }\end{array}$} & $\begin{array}{l}\text { Capital politique } \\
\text { et diplomatique } \\
\text { (opportunité d'un } \\
\text { rôle protagoniste) }\end{array}$ & $\begin{array}{l}\text { Difficile. Les bruits de communication entre le Brésil et } \\
\text { l'Arabie saoudite, l'Égypte et la Chine font ressortir des } \\
\text { relations tendues avec des pays extérieurs à l'axe Donald } \\
\text { Trump, affectant ainsi l'image du Brésil et sa tradition } \\
\text { d'engagement en faveur du multilatéralisme. La perte de } \\
\text { leadership dans la région sud-américaine est visible avec les } \\
\text { frictions entre le Brésil et l'Argentine, ainsi qu'avec la } \\
\text { résurgence d'un possible leadership mexicain sous } \\
\text { López Obrador, y compris dans le cas de la crise au Venezuela. } \\
\text { En outre, le gouvernement Bolsonaro présente une perte de } \\
\text { capital diplomatique dans le domaine de l'environnement, par } \\
\text { le biais d'un discours ultranationaliste et négationniste, un } \\
\text { discours qui a été mis à l'épreuve lors du désastre de } \\
\text { l'entreprise transnationale Vale à Brumadinho, dans le } \\
\text { Minas Gerais (25/01/2019). }\end{array}$ \\
\hline & $\begin{array}{l}\text { Perception } \\
\text { positive sur les } \\
\text { expertises du } \\
\text { Brésil en matière } \\
\text { de politiques } \\
\text { publiques }\end{array}$ & $\begin{array}{l}\text { Possible. Le Brésil présente une expertise en matière de } \\
\text { politiques publiques qu'il est toujours possible } \\
\text { d'internationaliser. Cependant, cette possibilité dépendra } \\
\text { directement de «l'image du Brésil » qui sera construite dans } \\
\text { les mois et les années à venir. Il est possible pour le Brésil de } \\
\text { continuer son action dans le domaine des opérations de paix } \\
\text { en partenariat avec l'ONU afin de satisfaire la branche } \\
\text { militaire du gouvernement. }\end{array}$ \\
\hline
\end{tabular}

\section{NOTES}

1. Blog de Ernesto Araújo, Metapolítica 17. Contra o globalismo, https:// www.metapoliticabrasil.com/ (consulté le 20 mars 2019).

\section{AUTEURS}

\section{RENATA ALBUQUERQUE RIBEIRO}

Docteure en science politique à l'Institut d'études sociales et politiques de l'Université de l'État de Rio de Janeiro (IESP-UERJ). Elle est également chercheuse au Laboratoire d'analyse politique mondiale du même institut (www.labmundo.org).

\section{CARLOS R. S. MILANI}

Professeur des universités à l'IESP-UERJ (www.iesp.uerj.br), directeur du LABMUNDO, ainsi que chercheur au CNPq-1C et à FAPERJ www.carlosmilani.com.br 